\title{
IMAGING OF DIRECTIONAL DISTRIBUTED NOISE SOURCES
}

\author{
Dimitri Papamoschou* \\ University of California, Irvine, Irvine, California 92697-3975
}

\begin{abstract}
This study relates to the imaging of noise sources that are distributed and strongly directional, such as in turbulent jets. The goal is to generate noise source maps that are self-consistent, i.e., their integration over the extent of the noise source region gives the far-field pressure autospectrum for a particular emission direction. This is possible by including a directivity factor in the formulation of the source cross-spectral density. The resulting source distribution is based on the complex coherence, rather than the cross-spectrum, of the measured acoustic field. For jet noise, which is not only directional but whose nature changes with emission angle, it is necessary to conduct the measurements with a narrow-aperture array. The resulting images must be devoid to the extent possible of spatial distortions caused by the array response. Two methods for obtaining clean images are addressed: deconvolution of the beamformer output and direct spectral estimation. The two techniques produced similar noise source maps for a Mach 0.9 cold jet.
\end{abstract}

\section{Nomenclature}

\begin{tabular}{|c|c|c|}
\hline$a$ & $=$ & ambient speed of sound \\
\hline$b$ & $=$ & beam width, $V(x, x \pm b / 2, \omega)=\frac{1}{2} V(x, x, \omega)$ \\
\hline$D_{j}$ & $=$ & jet diameter \\
\hline$f$ & $=$ & cyclic frequency \\
\hline$G_{m n}$ & $=$ & cross spectrum matrix \\
\hline$I_{\mathrm{ML}}$ & $=$ & integral of main lobe of point spread function \\
\hline $\mathcal{L}$ & $=$ & axial extent of noise source region \\
\hline$\ell_{m}(x)$ & $=$ & distance of microphone $m$ from source point $x$ \\
\hline$K$ & $=$ & number of discrete sources \\
\hline$M$ & $=$ & number of microphones \\
\hline$M_{j}$ & $=$ & jet Mach number \\
\hline$R$ & $=$ & array radius \\
\hline$q(x, \theta, t)$ & $=$ & noise source strength \\
\hline$S r$ & $=$ & Strouhal number $=f D_{j} / U_{j}$ \\
\hline$t$ & $=$ & time \\
\hline$T_{m n}$ & $=$ & array response matrix \\
\hline$U_{j}$ & $=$ & jet velocity \\
\hline$V(x, \xi \omega)$ & $=$ & point spread function \\
\hline$W_{m}, w_{m}$ & $=$ & weight for microphone $m$ \\
\hline $\bar{w}_{m}$ & $=$ & dimensionless weight for microphone $m$ \\
\hline$x, \xi$ & $=$ & spatial coordinates \\
\hline$\gamma_{m n}$ & $=$ & complex coherence \\
\hline$\theta$ & $=$ & polar angle from jet axis \\
\hline$\Theta_{m n}$ & $=$ & directivity matrix \\
\hline$\lambda$ & $=$ & wavelength \\
\hline$\Phi(x, \omega)$ & $=$ & array beamformer output \\
\hline$\psi(x, \omega)$ & $=$ & coherence-based source distribution \\
\hline$\Psi(x, \omega)$ & $=$ & auto-spectrum-based source distribution \\
\hline$\tau_{m}$ & $=$ & time delay for microphone $m$ \\
\hline
\end{tabular}

*Professor, Associate Fellow AIAA 


$\begin{array}{lll}\theta_{m} & = & \text { polar angle of microphone } m \\ \theta_{a} & = & \text { average array polar angle } \\ \omega & = & \text { radian frequency }=2 \pi f\end{array}$

\section{Introduction}

Noise source location is central to the understanding, modeling, and suppression of noise from aircraft. Microphone techniques have included acoustic mirrors, ${ }^{1}$ polar correlation, ${ }^{2}$ and phased arrays. ${ }^{3}$ The theoretical foundation of noise source location using cross-correlations of multiple microphone signals was established by Billingsley and Kinns. ${ }^{3}$ More recently, frequency-domain approaches for processing the microphone array data were introduced by Humphreys et al. ${ }^{4}$ For the aforementioned noise source imaging methods, the output of the instrument is a convolution between a known kernel (the point spread function) and the noise source distribution. The noise source distribution has a presumed form, e.g., an array of monopoles. Recent studies by Brooks and Humphreys ${ }^{9}$ and by Dougherty ${ }^{10}$ have proposed methods of deconvolution.

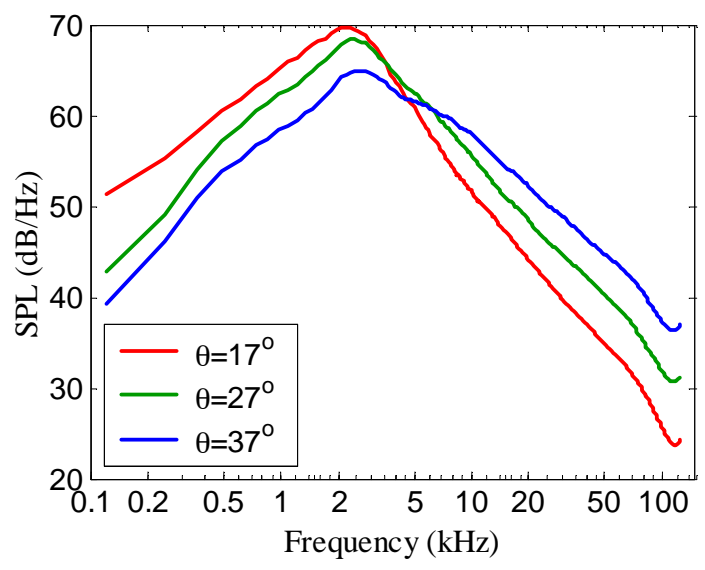

Fig.1 Variation of spectrum with polar angle from jet axis for a $M_{j}=0.9$ cold jet.

Imaging of jet noise sources is particularly challenging because the source is distributed and highly directional. Figure 1 illustrates the directionality of the spectra of a Mach 0.9 cold jet. Within a few degrees of polar angle the spectrum changes shape dramatically. Moreover, it is widely accepted that jet noise consists of two components with different nature: noise from large-scale turbulence in the direction of peak emission (aft quadrant); and noise from fine-scale turbulence in the broadside direction. ${ }^{5}$ This entails imaging of the noise source with a narrow-aperture array so that the two types of noise sources are not mixed up on a given array measurement. Although a significant number of studies have used microphone arrays to image jet noise, ${ }^{1,6-8}$ the array apertures were too large to distinguish between the two types of noise sources. Further, the effects of directivity were not addressed in a consistent manner. Recent work in our group used a small-aperture phased array to discriminate between large-scale and fine-scale noise sources. ${ }^{11}$ However, the resulting noise source maps were not self-consistent in the manner defined below.

In this paper we seek noise source imaging methods that produce self-consistent maps of the noise source distribution. This means that axial integration of the noise source gives the far-field auto-spectrum in a particular polar direction. It will be shown that directionality must be incorporated in the formulation of the noise source model. The resulting method is based on the coherence, rather than the cross-spectrum, of the pressure field. This idea represents an extension of concepts presented in the seminal paper on polar correlation technique by Fisher et al. ${ }^{2}$ In addition, we present two methods for improving the fidelity of imaging. The first method involves deconvolution of the beamforming output. The second approach is a direct spectral estimation method that obviates delay-and-sum beamforming. Finally we comment on the measured spatial coherence of the acoustic field and its proper interpretation. 


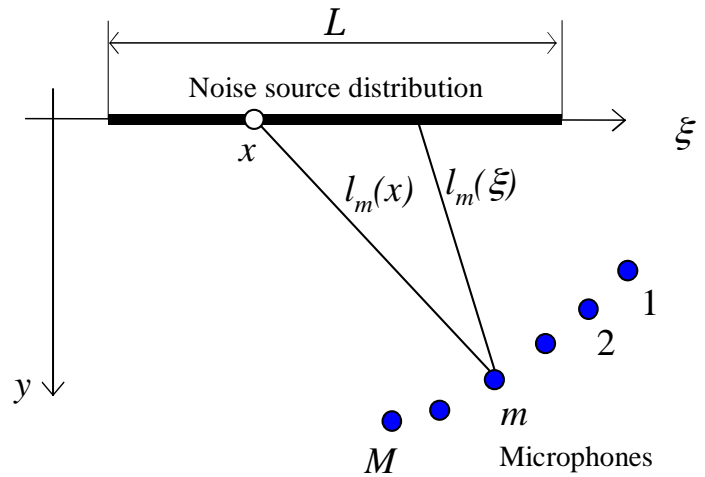

Fig.2 Linear distribution of noise sources and microphone array.

\section{Formulation of Directional Source Distribution}

Consider an axial distribution of directional sources, $q(x, \theta, t)$ and an array of $M$ microphones, as depicted in Fig. 2. At given $x$ the source amplitude varies with polar angle $\theta$ but the phase is constant on a sphere centered at the source. Assuming spherical spreading in a quiescent medium with uniform speed of sound $a$, the signal received by the $m^{t h}$ microphone is

$$
p_{m}(t)=\int_{\mathcal{L}} \frac{1}{\ell_{m}(x)} q\left(x, \theta_{m}(x), t-\tau_{m}(x)\right) d x
$$

where

$$
\tau_{m}(x)=\frac{\ell_{m}(x)}{a}
$$

is the retarded time from point $x$ to microphone $m$. Integration is carried over the region of interest $\mathcal{L}$ where significant sound sources are expected. The Fourier transform of the microphone output is

$$
P_{m}(\omega)=\int_{\mathcal{L}} \frac{1}{\ell_{m}(x)} e^{-i \omega \tau_{m}(x)} Q\left(x, \theta_{m}(x), \omega\right) d x
$$

In frequency-domain microphone array methods, the central parameter is the cross-spectral matrix

$$
G_{m n}(\omega) \equiv<P_{m}^{*}(\omega) P_{n}(\omega)>
$$

where $<>$ denotes time averaging. Substituting Eq. 3,

$$
G_{m n}(\omega)=\int_{\mathcal{L}} \int_{\mathcal{L}} \frac{1}{\ell_{m}(x) \ell_{n}(\xi)} e^{i \omega\left[\tau_{m}(x)-\tau_{n}(\xi)\right]}<Q^{*}\left(x, \theta_{m}(x), \omega\right) Q\left(\xi, \theta_{n}(\xi), \omega\right)>d x d \xi
$$

The bracketed term is the cross-spectral density of the noise source that needs to be modeled. We now assume an incoherent source distribution and, for reasons that will become apparent shortly, introduce a directivity matrix $\Theta_{m n}(x, \xi, \omega)$ as follows:

$$
<Q^{*}\left(x, \theta_{m}(x), \omega\right) Q\left(\xi, \theta_{n}(\xi), \omega\right)>=\psi(x, \omega) \Theta_{m n}(x, \xi, \omega) \delta(x-\xi)
$$

The resulting cross-spectral matrix is

$$
G_{m n}(\omega)=\int_{\mathcal{L}} \frac{1}{\ell_{m}(x) \ell_{n}(x)} e^{i \omega\left[\tau_{m}(x)-\tau_{n}(x)\right]} \psi(x, \omega) \Theta_{m n}(x, x, \omega) d x
$$

and its diagonal terms (auto-spectra) satisfy

$$
G_{m m}(\omega)=\int_{\mathcal{L}} \frac{1}{\ell_{m}(x)^{2}} \psi(x, \omega) \Theta_{m m}(x, x, \omega) d x
$$


The necessity of including the directivity matrix $\Theta_{m n}(x, \xi, \omega)$ in the formulation of the noise source is now clear. Without it, it is impossible for $\psi(x, \omega)$ to capture the directivity of the autospectra exemplified in Fig.1. A convenient form for the directivity matrix is

$$
\Theta_{m n}(x, \xi, \omega)=\sqrt{G_{m m}(\omega) G_{n n}(\omega)} \ell_{m}(x) \ell_{n}(\xi)
$$

Inclusion of the path lengths $\ell_{m}(x)$ and $\ell_{n}(\xi)$ makes $\Theta_{m n}$ a universal parameter, for a given jet, independent of microphone distances, provided that the microphones are in the acoustic far field. This formulation naturally brings out on the left hand side of Eq. 8 the complex coherence of the pressure field,

$$
\gamma_{m n}(\omega) \equiv \frac{G_{m n}(\omega)}{\sqrt{G_{m m}(\omega) G_{n n}(\omega)}}
$$

A similar formulation was proposed by Fisher et al. ${ }^{2}$ to account for the directivity of jet noise in the implementation of the polar correlation technique. Equation 8 thus takes the form

$$
\gamma_{m n}(\omega)=\int_{\mathcal{L}} e^{i \omega\left[\tau_{m}(x)-\tau_{n}(x)\right]} \psi(x, \omega) d x
$$

The diagonal terms satisfy

$$
1=\int_{\mathcal{L}} \psi(x, \omega) d x
$$

Once the coherence-based noise source distribution has been obtained, the auto-spectrum-based source distribution is calculated from

$$
\Psi\left(x, \omega, \theta_{m}\right)=\psi(x, \omega) \Theta_{m m}(x, x, \omega)=\psi(x, \omega) G_{m m}(\omega) \ell_{m}^{2}(x)
$$

For a given array position, Eq. 13 provides the noise source distribution corresponding to each microphone polar angle $\theta_{m}$. It is evident from Eqs. 8 and 13 that axial integration of $\Psi\left(x, \omega, \theta_{m}\right) / \ell_{m}(x)^{2}$ gives the auto-spectrum $G_{m m}(\omega)$ :

$$
\int_{\mathcal{L}} \frac{1}{\ell_{m}(x)^{2}} \Psi\left(x, \omega, \theta_{m}\right) d x=G_{m m}(\omega) \int_{\mathcal{L}} \psi(x, \omega) d x=G_{m m}(\omega)
$$

Therefore we have the desired self-consistent formulation for the directional noise source.

For the analysis that follows, it is convenient to introduce the array response matrix

$$
T_{m n}\left(x_{0}, \omega\right)=e^{i \omega\left[\tau_{m}\left(x_{0}\right)-\tau_{n}\left(x_{0}\right)\right]}
$$

It describes the modeled coherence of the acoustic field for a point source at $x=x_{0}$ (i.e., for $\psi(x)=\delta\left(x-x_{0}\right)$ ). Equation 11 then takes the form

$$
\gamma_{m n}(\omega)=\int_{\mathcal{L}} T_{m n}(x, \omega) \psi(x, \omega) d x
$$

\section{Methods for Noise Source Imaging}

In this section we discuss three methods for imaging the noise source: delay-and-sum beamforming based on the complex coherence (rather than on the cross-spectrum) of the acoustic field; deconvolution of the beamformer output; and direct spectral estimation using a minimization algorithm. The latter method obviates delay-and-sum beamforming. 


\section{A. Coherence-Based Beamforming}

In delay-and-sum beamforming, the array output is

$$
\Phi(x, \omega)=\sum_{m=1}^{M} \sum_{n=1}^{M} W_{m} W_{n} e^{i \omega\left[\tau_{n}(x)-\tau_{m}(x)\right]} G_{m n}(\omega)
$$

The microphone weights, $W_{m}$, are user-specified functions of $\omega$ and $x$. On selecting

$$
W_{m}=\frac{w_{m}(x, \omega)}{\sqrt{G_{m m}(\omega)}}
$$

and recognizing that the exponential term (steering matrix) is the complex transpose of the response matrix, Eq. 14, we obtain the coherence-based beamforming output

$$
\Phi(x, \omega)=\sum_{m=1}^{M} \sum_{n=1}^{M} w_{m} w_{n} T_{m n}^{*}(x, \omega) \gamma_{m n}(\omega)
$$

Expressing the coherence in terms of the source distribution, Eq. 15,

$$
\Phi(x, \omega)=\int_{\mathcal{L}} \sum_{m=1}^{M} \sum_{n=1}^{M} w_{m} w_{n} T_{m n}(\xi, \omega) T_{m n}^{*}(x, \omega) \psi(\xi, \omega) d \xi
$$

On defining the point-spread function (PSF) as

$$
V(x, \xi, \omega)=\sum_{m=1}^{M} \sum_{n=1}^{M} w_{m} w_{n} T_{m n}(\xi, \omega) T_{m n}^{*}(x, \omega)
$$

the coherence-based beamforming output becomes

$$
\Phi(x, \omega)=\int_{\mathcal{L}} \psi(\xi, \omega) V(x, \xi, \omega) d \xi
$$

Equation 20 shows that the array output is the convolution of the noise source distribution with the PSF. The PSF is not translation-invariant $(V(x, \xi, \omega) \neq V(x-\xi, \omega))$, so great care is needed to prevent spatial distortions of the apparent noise source because of the spatial dependence of the PSF. This is possible to first order by preserving the integral under the main lobe of the PSF. This integral is approximated here as the beam width $b(x, \omega)$ times the height of the point spread function:

$$
I_{\mathrm{ML}}=\int_{\text {main lobe }} V(x, \xi, \omega) d \xi \sim b(x, \omega) V(x, x, \omega)=D
$$

where $D$ is the jet diameter or any other characteristic length scale. From Eq. 19,

$$
V(x, x, \omega)=\sum_{m=1}^{M} \sum_{n=1}^{M} w_{m} w_{n}
$$

Therefore, the weights must satisfy

$$
w_{m}(x, \omega)=\bar{w}_{m} \sqrt{\frac{D}{b(x, \omega)}}
$$

where $\bar{w}_{m}$ are weight coefficients that satisfy $\sum \bar{w}_{m}=1$. The beamwidth $b(x, \omega)$ can be estimated analytically or computed numerically from Eq. 19. 


\section{B. Deconvolution}

Now we attempt to invert Eq. 20 and extract the coherence-based source distribution $\psi(x, \omega)$. From a mathematical standpoint, the weights $w_{m}$ are immaterial to this process as they cancel from both sides of Eq. 20. However, assigning weights of special forms may aid in the numerical implementation of the inversion process. At this point, for simplicity, we set $w_{m}=1$, in which case the beamforming output is

$$
\Phi(x, \omega)=\sum_{m=1}^{M} \sum_{n=1}^{M} T_{m n}^{*}(x, \omega) \gamma_{m n}(\omega)
$$

and the PSF is

$$
V(x, \xi, \omega)=\sum_{m=1}^{M} \sum_{n=1}^{M} T_{m n}(\xi, \omega) T_{m n}^{*}(x, \omega)
$$

For a given frequency, the integral of Eq. 20 can be expressed as a summation over a finite number of sources $K$. Applying the discretizations,

$$
\begin{aligned}
\Phi(x, \omega) & \rightarrow \Phi_{i} \\
V(x, \xi, \omega) & \rightarrow V_{k i} \\
\psi(\xi, \omega) \Delta \xi & \rightarrow \psi_{k}
\end{aligned}
$$

we obtain the linear system

$$
\Phi_{i}=\sum_{k=1}^{K} V_{i k} \psi_{k}
$$

The solution procedure generally follows the DAMAS approach of Brooks and Humphreys. ${ }^{9}$ One distinction, however, is that we use the Richardson-Lucy (RL) inversion algorithm ${ }^{14,15}$ which has found wide application in image restoration. The advantage of RL over Gauss-Seide ${ }^{9}$ is that its output is inherently non-negative and does not depend on the sequence of the equations. The iteration algorithm is

$$
\begin{aligned}
\psi_{k}^{(j)} & =\psi_{k}^{(j-1)} \frac{1}{\sum_{i=1}^{K} V_{i k}} \sum_{i=1}^{K} \frac{V_{i k} \Phi_{i}}{\tilde{\Phi}_{i}} \\
\tilde{\Phi}_{i} & =\sum_{k=1}^{K} V_{i k} \psi_{k}^{(j-1)}
\end{aligned}
$$

The spatial extent of the region investigated and the resolution of grid points are critical parameters for the success of the deconvolution scheme. Important guidance comes from the deconvolution work of Brooks and Humphreys. ${ }^{9}$ As shown in Fig. 3, the investigation domain consists of two overlapping regions: the scan region over which Eq. 27 is applied and the region of interest that includes the relevant noise sources. The scan region is larger than or equal to the region of interest. The length of the scan region $\Lambda$ should be greater than the beam width and was selected to be $\Lambda=2 b$. For the present array it was determined that $b \approx 2 \lambda$, therefore $\Lambda=4 \lambda$. The region of interest was chosen as $-5 D<x<25 D$, so its length was $\mathcal{L}=30 D$. The scan region was arranged symmetrically over the region of interest, as shown in Fig. 3. The spatial resolution should be a fraction of the wavelength and was set at $\Delta \xi=0.25 \lambda$. However, at low frequencies where the wavelength is very large, the resolution was set at $\Delta \xi=0.01 \mathcal{L}$. The combined scheme for the resolution was

$$
\Delta \xi=\min (0.01 \mathcal{L}, 0.25 \lambda)
$$

Typically, the method converged to a residual of less than 0.05 in 50 iterations, with slow improvement thereafter. The number of iterations was set at 200 . 


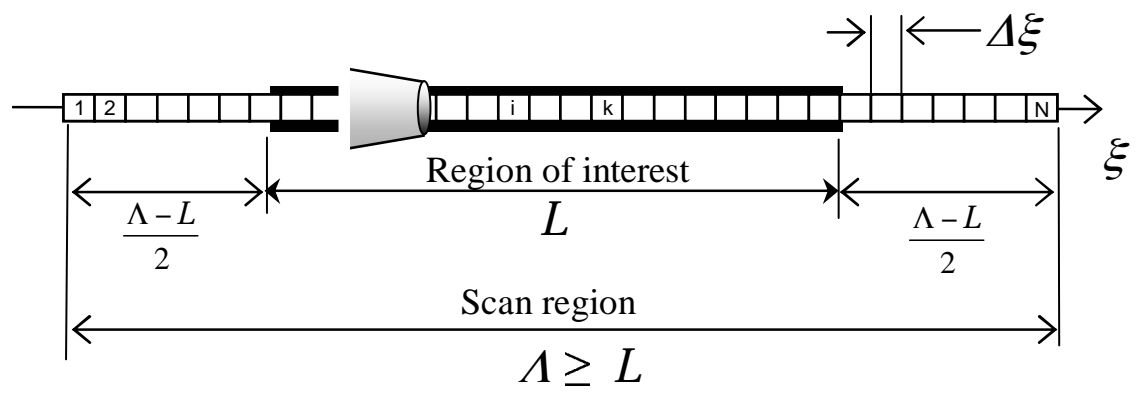

Fig.3 Scan region and region of interest for deconvolution algorithm.

\section{Direct Spectral Estimation}

An alternative to beamforming followed by deconvolution is direct estimation of the source distribution from Eq. 15

$$
\gamma_{m n}(\omega)=\int_{\mathcal{L}} T_{m n}(x, \omega) \psi(x, \omega) d x
$$

The left- and right-hand sides represent the measured and modeled coherence matrices, respectively. We seek a source distribution $\psi(x, \omega)$ that minimizes the error between these two matrices. Here we do not manipulate the phase of each signal to steer the array in a particular direction. Instead, for each frequency, we minimize the errors between the independent elements of the measured and modeled coherence matrices. For $M$ microphones, there are $\left(M^{2}-M\right) / 2$ distinct off-diagonal elements and 1 distinct diagonal element. The off-diagonal elements contain real and imaginary parts, making the total number of independent values to be minimized

$$
J=M^{2}-M+1
$$

which equals 57 for $M=8$. Letting $j$ be the index of the distinct real and imaginary values of $\gamma_{m n}$, we write Eq. 15 as follows:

$$
\gamma_{j}(\omega)=\int_{\mathcal{L}} \psi(x, \omega) T_{j}(x, \omega) d x
$$

Upon the discretizations

$$
\begin{gathered}
\psi(x, \omega) \delta x \rightarrow \psi_{k} \\
T_{j}(x, \omega) \rightarrow T_{j k}
\end{gathered}
$$

we have have the following linear system for each frequency:

$$
\gamma_{j}=\sum_{k=1}^{K} \psi_{k} T_{j k}
$$

To proceed further we adopt the approach Blacodon and Elias ${ }^{16}$ who addressed a similar situation with airframe noise sources. Given that negative sources are not physical, a non-negative constraint is added by expressing the sources as

$$
\psi_{k}=a_{k}^{2}
$$

This leads to the least-squares unconstrained minimization of the function

$$
F\left(a_{k}\right)=\sum_{j=1}^{J}\left|\gamma_{j}-\sum_{k=1}^{K} a_{k}^{2} T_{j k}\right|^{2}
$$

The minimization is done iteratively using the restarted conjugate gradient method of Shanno and Phua. ${ }^{17}$ The number of noise sources $K$ is generally arbitrary and does not need to match the number of independent equations $J$. However, the best results are obtained when $K \approx J$. For the results in this paper, $K=J=57$, and the sources were obtained on a fixed $x$-vector for all frequencies. The error was quantified in terms of 
$\sqrt{F /\left\|\gamma_{j}\right\|}$ and typically was on the order of 0.1 . The error in the diagonal terms was much smaller and around 0.01 .

From Eqs. 19 and 20 we recognize that the deconvolution integral amounts to the Frobenius inner product of the direct spectral estimation relation, Eq. 15, with the steering matrix $T_{m n}^{*}(x, \omega)$. In fact, one could generalize the deconvolution problem by taking the inner product of Eq. 15 with any suitable matrix that would facilitate the inversion.

The direct spectral estimation method is computationally more expensive than the deconvolution because the conjugate gradient method searches in $K$ directions to minimize the gradient. Typically the routine required about 20 function calls to for the gradient to be minimized. Eq. 12 was satisfied to within 5\%. On the other hand, the method is attractive because of its directness and simple formulation which will facilitate the incorporation of more advanced models for the noise source (e.g. sources with finite coherence length scales).

\section{Experimental Setup}

\section{A. Flow facility}

Experiments were conducted in UCI's Jet Aeroacoustics Facility, described in earlier publications. ${ }^{12}$ The facility was operated in single-stream mode with pure air, at ambient reservoir temperature, supplied to a convergent round nozzle with exit diameter $D_{j}=21.8 \mathrm{~mm}$. The nozzle pressure ratio was 1.69 resulting in exit Mach number $M_{j}=0.9$ and exit velocity $U_{j}=287 \mathrm{~m} / \mathrm{s}$. The jet Reynolds number was $5 \times 10^{5}$.

\section{B. Microphone Array}

The microphone phased array consists of eight 3.2-mm condenser microphones (Brüel \& Kjær Model 4138) arranged on a circular arc centered at the vicinity of the nozzle exit. Figure 4 shows the array geometry. The polar aperture of the array for this experiment was $30^{\circ}$ and the array radius was $1 \mathrm{~m}$. The angular spacing of the microphones was logarithmic, starting from $2^{\circ}$ for microphones 1 and 2 and ending with $10^{\circ}$ for microphones 7 and 8. Uneven microphone spacing was used to mitigate the effects of spatial aliasing. The entire array structure is rotated around its center to place the array at the desired observation angle. Positioning of the array is done remotely using a stepper motor. An electronic inclinometer displays the position of first microphone. The distances between the centers of the microphone grids were measured with accuracy of $0.1 \mathrm{~mm}$ using a digital caliper. A geometric calibration procedure provided the position of each mirophone relative to the nozzle exit with accuracy of $2 \mathrm{~mm}$.

The arrangement of the microphones inside the anechoic chamber, and the principal electronic components, are shown in Fig. 4. The microphones were connected, in groups of four, to two amplifier/signal conditioners (Brüel \& Kjær Nexus 2690-A-OS4) with low-pass filter set at $300 \mathrm{~Hz}$ and high-pass filter set at $100 \mathrm{kHz}$. The four-channel output of each amplifier was sampled at $250 \mathrm{kHz}$ per channel by a multi-function data acquisition board (National Instruments PCI-6070E). Two such boards, one for each amplifier, were installed in a Pentium 4 personal computer. National Instruments LabView software was used to acquire the signals. Time delays due to multiplexing in the data acquisition boards were measured by feeding the same signal to all the channels. These delays were compensated for in the source imaging algorithms.

The array observation angle defined as $\theta_{a}=\left(\theta_{1}+\theta_{8}\right) / 2$. This paper discusses results obtained at two array observation angles: $\theta_{a}=30^{\circ}$ and $\theta_{a}=105^{\circ}$. They correspond to noise generated by large-scale and fine-scale turbulence, respectively. The placement of the microphones for each observation angle is plotted in Fig. 5 . 


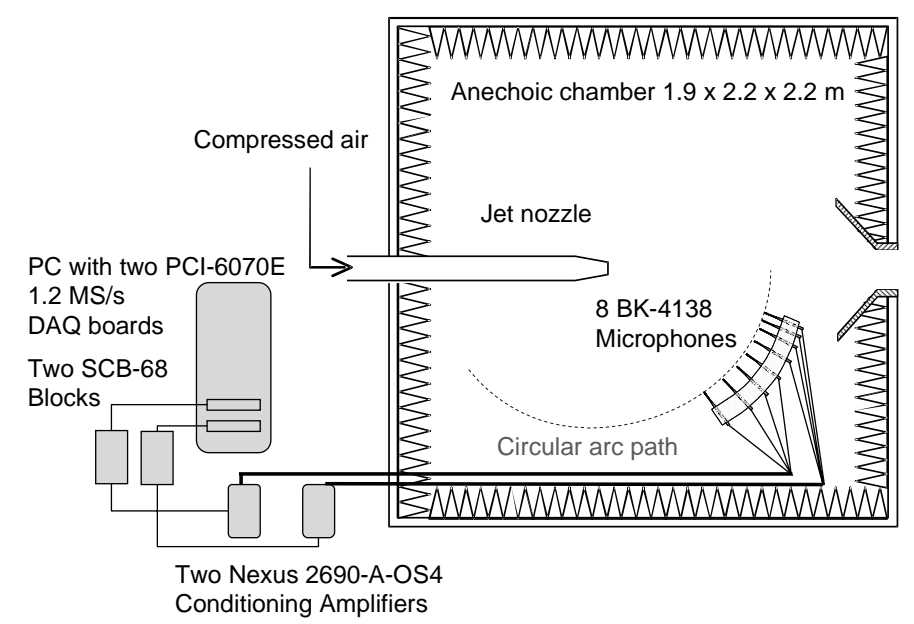

Fig.4 Primary components of microphone array system.

a)

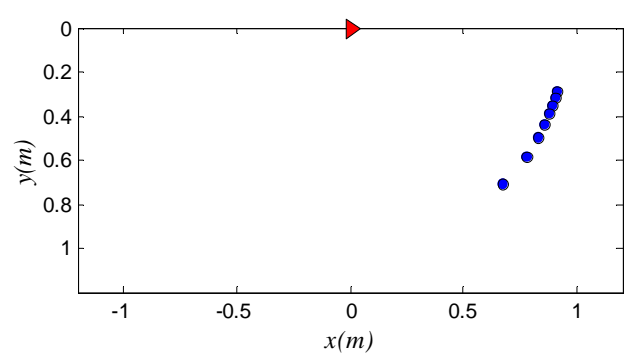

b)

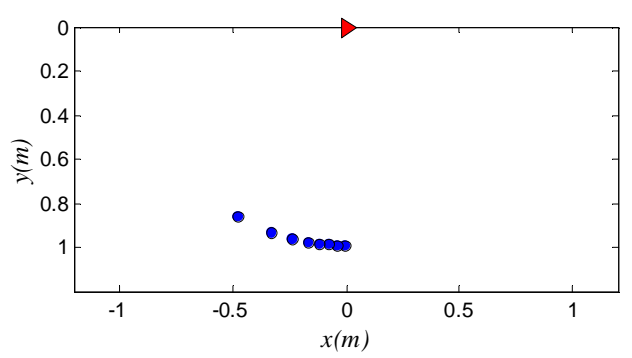

Fig.5 Two positions of array: a) $\theta_{a}=30^{\circ}$; b) $\theta_{a}=105^{\circ}$. Triangles indicate nozzle exit.

\section{Data Processing}

The computation of the cross spectrum matrix, Eq. 4, involved the following steps. Each microphone signal consisted of $N_{s}=2^{18}=262144$ samples acquired at a sampling rate $F_{s}=250 \mathrm{kHz}$. The maximum resolvable (Nyquist) frequency was $F_{s} / 2=125 \mathrm{kHz}$, although the high-pass filter was set a little lower at $f=100 \mathrm{kHz}$. The size of the Fast Fourier Transform was $N_{\mathrm{FFT}}=2024$ yielding a frequency resolution of $122 \mathrm{~Hz}$. Each signal was divided into $K=64$ blocks of 4096 samples each, and the data within each block was windowed using a Hamming window. The cross-spectrum matrix $G_{m n}^{k}$ for block $k$ was computed using Fortran routines for autospectra and crossspectra. The total cross-spectrum matrix was obtained from

$$
G_{m n}(f)=\frac{1}{K W_{h}} \sum_{k=1}^{K} G_{m n}^{k}(f)
$$

where $W_{h}$ is a weighting constant for the Hamming window. Since the cross-spectrum matrix is Hermitian, only the diagonal and upper-triangle elements were computed; the lower-triangle elements were calculated as complex conjugates of the upper-triangle elements.

In calculating the auto-spectrum based source distribution, Eq. 13, the autospectrum $G_{m m}$ undergoes corrections to render it in lossless form. These corrections are performed in units of decibels:

$$
10 \log _{10}\left[G_{m m}(\omega)\right]=10 \log _{10}\left[G_{m m_{\text {raw }}}(\omega)\right]+93.98-C_{\mathrm{fr}}(f)-C_{\mathrm{ff}}(f)+\alpha(f) R_{m}
$$

The constant 93.98 comes from the normalization of the pressure by the reference pressure of $20 \mu \mathrm{Pa}$, that is, $-20 \log _{10}\left(20 \times 10^{-6}\right)=93.98$. $C_{\mathrm{fr}}$ and $C_{\mathrm{ff}}$ are the corrections for the actuator response and free-field 
response, respectively; they are based on data provided by the manufacturer of the microphone and are practically the same for all the microphones. $\alpha$ is the atmospheric absorption coefficient $(\mathrm{dB} / \mathrm{m})$, computed using the formulas proposed by Bass et al. ${ }^{13}$ for the measured values of relative humidity and temperature of the ambient air.

\section{Results}

\section{A. Noise Source Maps}

We start with presentation of maps of the coherence-based source distributions $\psi(x, \omega)$ as viewed from the two array angles of Fig. 5. Figure 6 shows the source distributions based on delay-and-sum beamforming. The noise maps are subject to blurring and distortions from the point spread function. It will be seen that difference in the source map at $\theta_{a}=30^{\circ}$ and $\theta_{a}=105^{\circ}$ are due mainly to the variance in PSF between the two o

a)
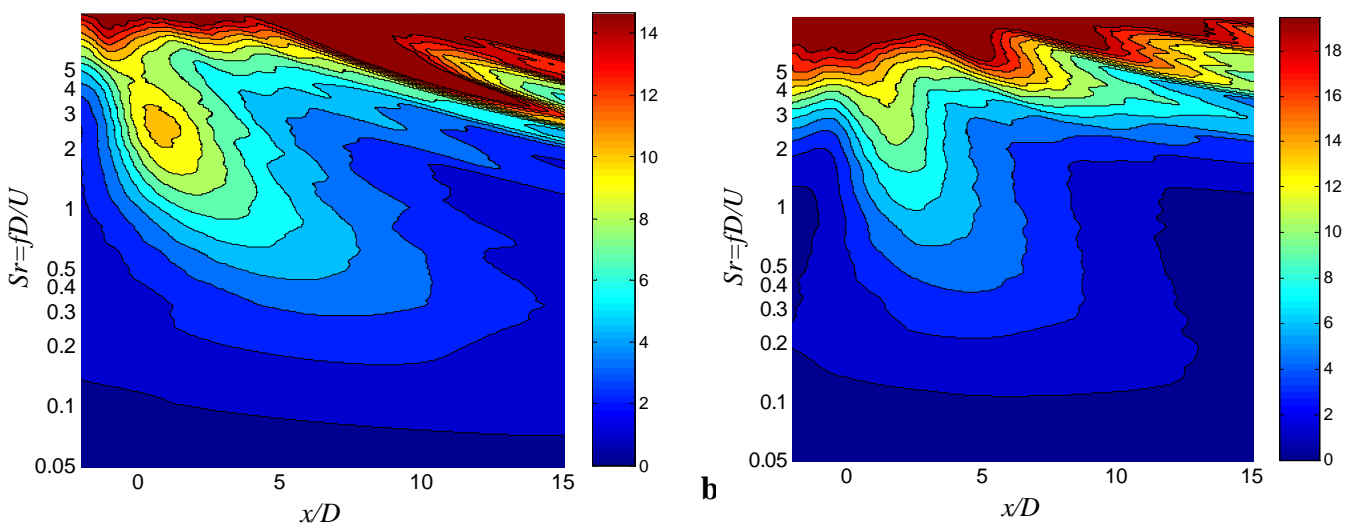

Fig.6 Coherence-based beamforming output (linear scale). a) $\theta_{a}=30^{\circ}$; b) $\theta_{a}=105^{\circ}$
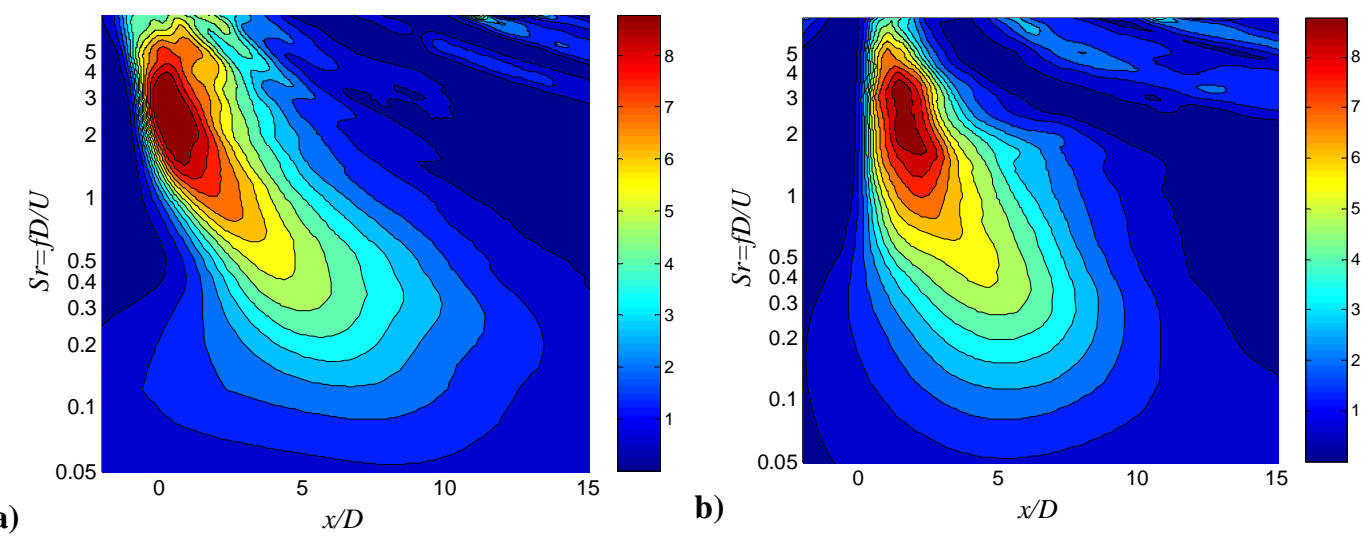

Fig.7 Coherence-based source distribution after deconvolution of beamforming output (linear scale). a) $\theta_{a}=30^{\circ}$; b) $\theta_{a}=105^{\circ}$

Figure 7 shows the corresponding source maps after RL deconvolution. The results are now much clearer and confined, and do not show major differences between the two array angles. This means that, in terms of coherence, the distributions of large-scale and fine-scale noise sources are quite similar. The results of 

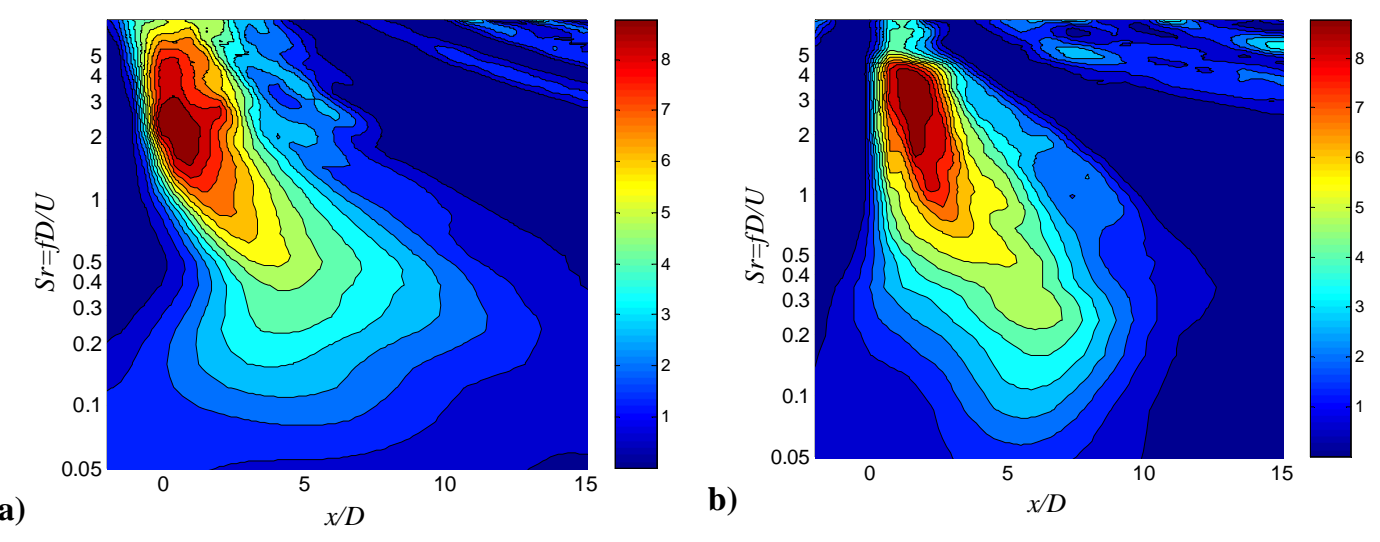

Fig.8 Coherence-based source distribution obtained by direct spectral estimation (linear scale). a) $\theta_{a}=30^{\circ}$; b) $\theta_{a}=105^{\circ}$

the direct spectral estimation are shown in Fig. 8. They agree well with the results obtained by the RL deconvolution in Fig. 7. This should come as no surprise since, as mentioned earlier, the deconvolution simply amounts the inner product of the direct spectral estimation relation with the array steering matrix. Nevertheless, the similarity in the results of the two methods inspires confidence that both methods are producing reasonable noise source maps.

For both the deconvolution and direct spectal estimation methods, the noise source maps are clean up to about $S r=3$, above which weak "ghost" images appear. The appearance of ghost images are very sensitive to the microphone positions. Deviations from their assumed positions of as little as $0.1 \mathrm{~mm}$ can create ghost images at high frequency. Iterative schemes to minimize the ghost images by small perturbations of the assumed microphone positions are under development. Therefore, for $S r>3$ the levels in the main map start becoming inaccurate because the axial integration in Eq. 20 (deconvolution) or Eq. 15 (direct spectral estimation) includes the ghost images. The expectation is that the intensity of the noise sources should increase as their extent becomes smaller with increasing frequency.

Now we examine the autospectrum-based source distribution, obtained by Eq 13. Shown in Fig.9 are results for $\theta=24^{\circ}$ obtained via the three methods. The beamforming produces an axially-spread-out image of the noise sources. The improvement in resolution with the RL deconvolution and direct spectral estimation methods is notable.
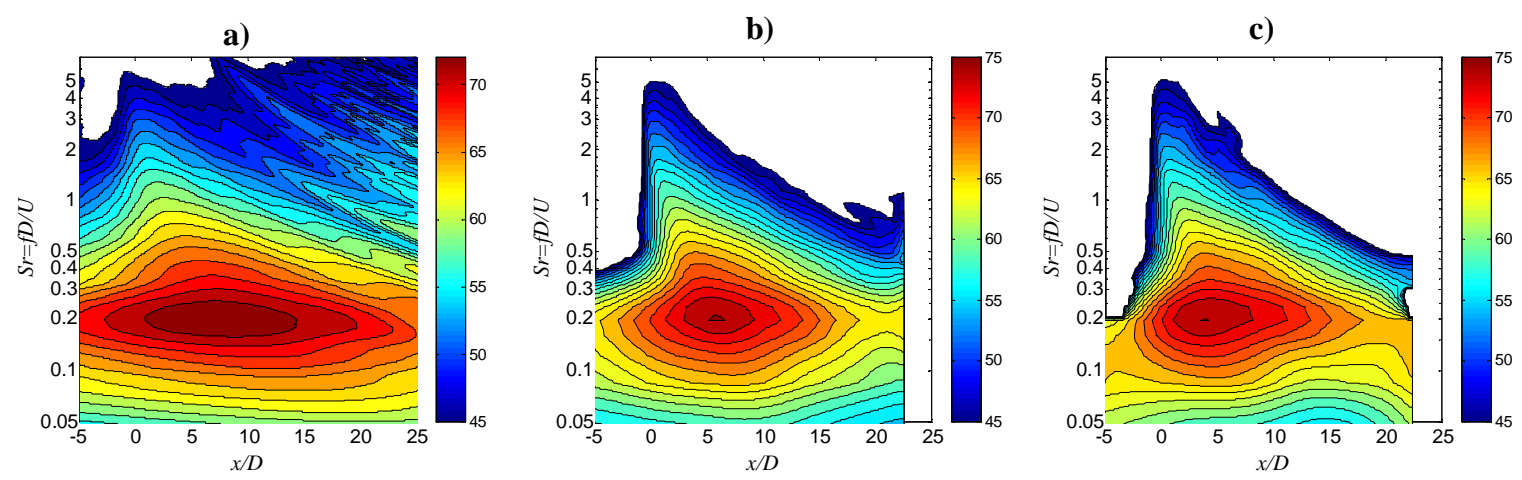

Fig.9 Autospectrum-based source distribution for $\theta=24^{\circ}$ (decibels). a) Beamforming; b) RL deconvolution; c) direct spectral estimation. 


\section{B. Spatial Coherence of Acoustic Field}

The results of Fig.7 show a strong similarity between noise sources emitting at small angles and large angles relative to the jet axis. This may be surprising given recent studies that infer strong spatial coherence for noise sources emitting at small angles and little spatial coherence for noise sources emitting at large angles. ${ }^{18,19}$ The assessment of those studies was based on the spatial coherence of the acoustic far field. It will be shown here that it is critical to account for the response of the instrument that images the noise source when connecting the spatial coherence of the acoustic field to the spatial coherence of the source.

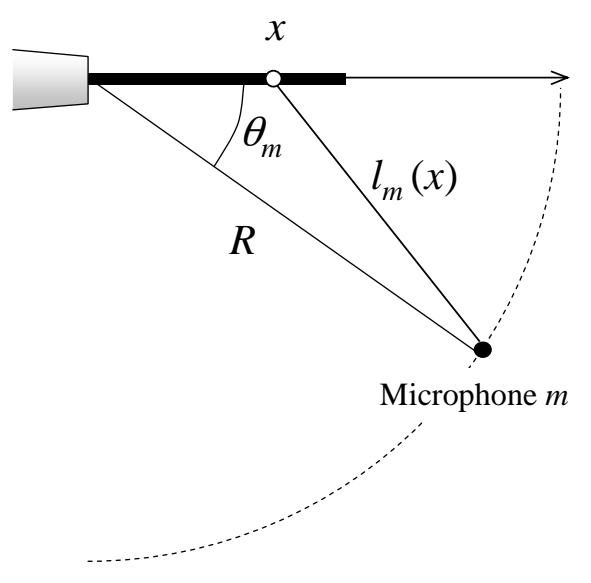

Fig.10 Geometry of polar arrangement of microphones

The response of the imaging instrument - in this case the microphone array - is a function not only of the arrangement of the microphones but also of the model for the acoustic source. For the spatially-incoherent source model used in Section II, the response of the array is given by Eq. 14. In terms of the path lengths $\ell$, it takes the form

$$
T_{m n}(x, \omega)=e^{i(\omega / a)\left[\ell_{m}(x)-\ell_{n}(x)\right]}
$$

Consider the polar arrangement of microphone of Fig. 10. From the geometry,

$$
\ell_{m}(x)^{2}=R^{2}-2 R x \cos \theta_{m}+x^{2}
$$

For $R>>x$, we can make the following approximations

$$
\ell_{m}(x)-\ell_{n}(x)=\frac{\ell_{m}(x)^{2}-\ell_{n}(x)^{2}}{\ell_{n}(x)+\ell_{m}(x)} \approx \frac{2 R x\left(\cos \theta_{n}-\cos \theta_{m}\right)}{2 R}=x\left(\cos \theta_{n}-\cos \theta_{m}\right)
$$

This can further be written as

$$
\ell_{m}(x)-\ell_{n}(x)=x 2 \sin \left(\frac{\theta_{m}-\theta_{n}}{2}\right) \sin \left(\frac{\theta_{m}+\theta_{n}}{2}\right)
$$

and shows that the response of the microphone array depends not only on the separation angle $\Delta \theta=\theta_{n}-\theta_{m}$ but also on the average angle $\left(\theta_{m}+\theta_{n}\right) / 2$ of any pair of microphones. The latter term causes a slow response with $\Delta \theta$ at shallow polar angles and a fast response at polar angles near $90 \mathrm{deg}$.

Figure 12 plots the coherence modulus $\left|\gamma_{m n}\right|$ versus angular separation $\theta_{n}-\theta_{m}$ and versus the array angular response parameter $2 \sin \left[\left(\theta_{m}-\theta_{n}\right) / 2\right] \sin \left[\left(\theta_{m}+\theta_{n}\right) / 2\right]$ for the two array polar angles and for two Strouhal numbers. All the non-trivial permutations of $\theta_{m}$ and $\theta_{n}$ are included. The coherence versus separation plots show the trends seen by previous investigators, namely, the coherence decays much faster with $\Delta \theta$ at large observation angles with respect to the jet axis than at shallow angles. However, when the coherence 
is plotted versus the array angular response parameter it is seen that the distributions for the two array observation angles practically collapse.

This exercise shows that an axial source distribution modeled as having the same spatial coherence length scale (in this case zero) in all directions creates an acoustic field with spatial (polar) correlation that depends on the observation angle. This is simply a geometric effect that can be "filtered-out" by accounting for the response of the array. This step is naturally included in the deconvolution and direct spectral estimation processes, hence the similarity of the source distributions in Figs. 7a and 7b, and in Figs. 8a and 8b. For sources with finite correlation length scale the response becomes more complex. However, there is little doubt that the geometric effects would act in the same basic manner as for the incoherent source distribution.
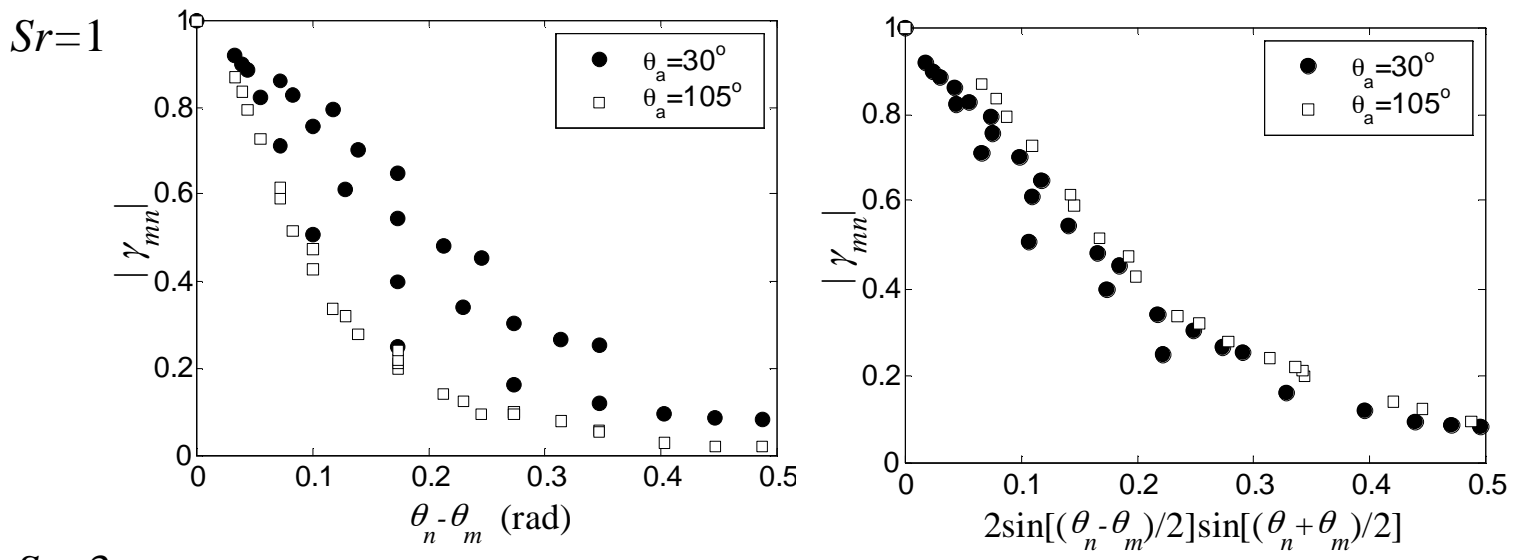

$\mathrm{Sr}=3$
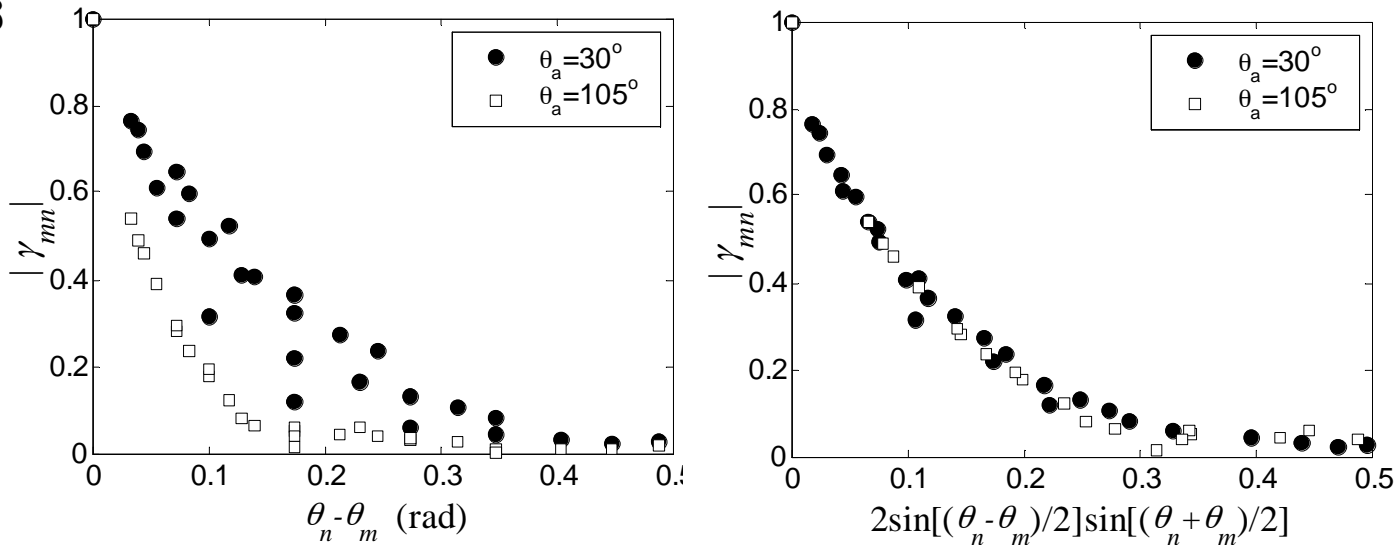

Fig.11 Coherence magnitude of acoustic field versus angular separation and versus array angular response parameter for two Strouhal numbers.

\section{Conclusions}

The paper presents a formulation of directive noise sources for microphone array methods. The goal is to generate noise source maps that are self-consistent, i.e., their integration over the extent of the noise source region gives the far-field pressure autospectrum for a particular emission direction. This is possible by including a directivity factor in the formulation of the source cross-spectral density. The resulting source distribution is based on the complex coherence, rather than the cross-spectrum, of the measured acoustic field. For jet noise, which is not only directional but whose nature changes with emission angle, it is necessary to conduct the measurements with a narrow-aperture array. 
The resulting images need to be processed to remove as much as possible the spatial distortions introduced by the array response. Two such methods are addressed here, a deconvolution technique based on the Richardson-Lucy inversion and a direct spectral estimation method that obviates delay-and-sum beamforming. Both approaches enhanced significantly the spatial resolution of the noise source maps and provided similar results. It is shown that the deconvolution integral amounts to the tensor inner product of the direct spectral estimation relation with the array response matrix.

Finally, it is shown that the measured spatial coherence of the acoustic field must be interpreted carefully to make any assessment about the spatial coherence of the noise source. In particular, the response of the microphone array, which depends on observation angle, must be accounted for in such assessment.

\section{References}

${ }^{1}$ Laufer, J., Schlinker, R., and Kaplan, R.E., "Experiments on Supersonic Jet Noise," AIAA Journal, Vol. 14, No.4, 1976, pp. 489-497.

${ }^{2}$ Fisher, M.J., Harper-Bourne, M., and Glegg, S.A.L., "Jet Engine Source Location: The Polar Correlation Technique," Journal of Sound and Vibration, Vol. 51, No.1, 1977, pp. 23-54.

${ }^{3}$ Billingsley, J. and Kinns, R., "The Acoustic Telescope," Journal of Sound and Vibration, Vol. 48, No.4, 1976, pp. 485-510.

${ }^{4}$ Humphreys, W.M., Brooks, T.F., Hunter, W.W., and Meadows, K.R., "Design and Use of Microphone Directional Arrays for Aeroacoustic Measurements," AIAA-98-0471, Jan. 1998.

${ }^{5}$ Tam, C.K.W., "Jet Noise: Since 1952," Theoretical and Computational Fluid Dynamics, Vol. 10, 1998, pp. 393-405.

${ }^{6}$ Narayanan, S., Barber, T.J., and Polak, D.R., "High Subsonic Jet Experiments: Turbulence and Noise Generation Studies," AIAA Journal, Vol. 40, No. 3, 2002, pp. 430-437.

${ }^{7}$ Lee, S.S. and Bridges, J., "Phased-array Measurements of Single Flow Hot Jets," AIAA-2005-2842, May 2005 .

${ }^{8}$ Venkatesh S.R., Polak D.R., and Narayanan S., "Beamforming Algorithm for Distributed Source Localization and its Application to Jet Noise," AIAA Journal, Vol. 41, No.7, 2003, pp. 1238-1246.

${ }^{9}$ Brooks, T.F., and Humphreys, W.M., "A Deconvolution Approach for the Mapping of Acoustic Sources (DAMAS) Determined from Phased Microphone Arrays," AIAA-2004-2954, May 2004.

${ }^{10}$ Dougherty, R.P., "Extensions of DAMAS and Benefits and Limitations of Deconvolution in Beamforming," AIAA-2005-2961, May 2005.

${ }^{11}$ Papamoschou, D., and Dadvar, A., "Localization of Multiple Types of Jet Noise Sources," AIAA Paper 2006-2644, May 2006.

${ }^{12}$ Papamoschou, D. and Debiasi, M., "Directional Suppression of Noise from a High-Speed Jet," AIAA Journal, Vol. 39, No. 3, 2001, pp. 380-387.

${ }^{13}$ Bass, H.E., Sutherland, L.C., Blackstock, D.T., and Hester, D.M., "Atmospheric Absorption of Sound: Further Developments," Journal of the Acoustical Society of America, Vol. 97, No. 1, 1995, pp. 680-683.

${ }^{14}$ Richardson, W.H., "Bayesian-Based Iterative Method of Image Restoration," Journal of the Optical Society of America, Vol. 62, No. 1, 1972, pp. 55-

${ }^{15}$ Lucy, L.B., "Iterative Technique for Rectification of Observed Distribution," Astronomical Journal, Vol. 79, No. 6, 1964, pp. 745-754. 
${ }^{16}$ Blacodon, D., and Elias, G., "Level Estimation of Extended Acoustic Sources Using a Parametric Method," Journal of Aircraft, Vol. 41, No. 6, 2004, 1360-1369.

${ }^{17}$ Shanno, D.F., and Phua, K.H., "Minimization of Unconstrained Multivariate Functions," ACM Transactions on Mathematical Software, Vol.6, No.4, 1980, pp. 618-622.

${ }^{18}$ Viswanathan, K., "Investigation of the Sources of Jet Noise," AIAA Paper 2007-3601, May 2007.

${ }^{19}$ Tam, C.K.W., Viswanathan, K., Ahuja, K.K., and Panda, J., "The Sources of Jet Noise: Experimental Evidence," AIAA Paper 2007-3641, May 2007. 